INPLASY

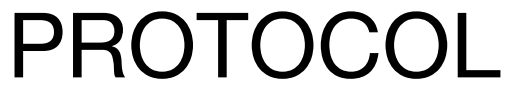

To cite: Liu et al. Effects of warm needle acupuncture on Temporomandibular Joint

Disorders: A systematic review and meta-analysis of randomized controlled trials. Inplasy protocol 202160030. doi:

10.37766/inplasy2021.6.0030

Received: 09 June 2021

Published: 09 June 2021

Corresponding author:

Taipeng Tan

tanyizhen12345@126.com

Author Affiliation:

Acupuncture department, Heilongjiang Provincial

Academy of Traditional

Chinese Medicine

Sciences,Harbin, China; No. 142, Sanfu Street

Support: National Training Project.

Review Stage at time of this submission: Piloting of the study selection process.

\section{Effects of warm needle acupuncture on Temporomandibular Joint Disorders: A systematic review and meta-analysis of randomized controlled trials}

Liu, GF1; Gao, Z2; Liu, ZN3; Yang, M4; Zhang, S5; Tan, TP6.

Review question / Objective: Effects of warm needle acupuncture on Temporomandibular Joint Disorders: A systematic review and meta-analysis of randomized controlled trials.

Condition being studied: Temporomandibular joint disorders(TMD)is a common and intractable disease in stomatology with the main symptoms of pain in the temporomandibular joint area, joint murmurs, and mandibular movement disorders, which seriously affect the work and life of patients. The disease mostly occurs in young and middleaged, and the prevalence increases with age. In recent years, there have been more and more researches on the treatment of TMD with warm needle acupuncture(WNA), and the quality of research has gradually improved. This study aims to use a systematic review method to understand the efficacy of WNA in the treatment of TMD.

INPLASY registration number: This protocol was registered with the International Platform of Registered Systematic Review and Meta-Analysis Protocols (INPLASY) on 09 June 2021 and was last updated on 09 June 2021 (registration number INPLASY202160030).

Conflicts of interest:

None declared.

\section{INTRODUCTION}

Review question / Objective: Effects of warm needle acupuncture on Temporomandibular Joint Disorders: A systematic review and meta-analysis of randomized controlled trials.

Condition being studied: Temporomandibular joint disorders (TMD) is a 
common and intractable disease in stomatology with the main symptoms of pain in the temporomandibular joint area, joint murmurs, and mandibular movement disorders, which seriously affect the work and life of patients. The disease mostly occurs in young and middle-aged, and the prevalence increases with age. In recent years, there have been more and more researches on the treatment of TMD with warm needle acupuncture(WNA), and the quality of research has gradually improved. This study aims to use a systematic review method to understand the efficacy of WNA in the treatment of TMD.

\section{METHODS}

Participant or population: Patients clearly diagnosed with temporomandibular joint disorder.

Intervention: Warm needle acupuncture was the main intervention.

Comparator: Acupuncture, Western medicine or some other means of treatment.

Study designs to be included: Randomised controlled trial will be included.

Eligibility criteria: Patients clearly diagnosed with temporomandibular joint disorder, age, gender, course of disease and other factors are not limited.

Information sources: We will search the randomized controlled trails (RCT) literatures of WNA for TMD in 9 electronic databases, including 5 English databases [PubMed, EMBASE, the Cochrane Central Register of Controlled Trials (Cochrane Library), Web of Science,and MEDLINE] and 4 Chinese databases [Chinese National Knowledge Infrastructure(CNKI), Chinese VIP Information, Wanfang Database, and Chinese Biomedical Literature Database (CBM)],from their inception to May 2021, to identify and retrieve all randomised controlled trials, describing the use of WNA for treatment of TMD. We also contacted the authors, searching for experimental data, clinical research registration, or other related grey literature.

Main outcome(s): Tenderness, ringing, chewing function of Temporomandibular Joint Disorders which associated to effective rate and cure rate.

Quality assessment / Risk of bias analysis: Two reviewers will independently assesses the quality of the selected studies according to the Cochrane Collaboration's tool for randomized controlled trials. Items will be evaluated in three categories: Low risk of bias, unclear bias and high risk of bias. The following characteristics will be evaluated: Random sequence generation (selection Bias)Allocation concealment (selection bias) Blinding of participants and personnel ((performance bias) Incomplete outcome data (attrition bias)Selective reporting (reporting bias) Other biases Results from these questions will be graphed and assessed using Review Manager 5.3.

Strategy of data synthesis: Two authors will independently extract data. Any disagreement will be resolved by discussion until consensus is reached or by consulting a third author. The following data will be extracted: author, year of publication, country where the study was conducted, study period, original inclusion criteria, total number of people included in the study, group, interventions, sample size, age (years), gender, course of the disease, course of treatment.

Subgroup analysis: Once individual studies may consist of multiple treatment group, subgroup analysis will be performed to explain heterogeneity if possible. Factors such as following will be considered: Patients characteristics (age, sex), Duration and frequency of therapy.

Sensitivity analysis: In order to ensure the stability of the outcome index results, the sensitivity analysis of each outcome index was carried out.

Country(ies) involved: China. 
Keywords: Temporomandibular Joint Disorders, warm needle acupuncture, RCT, systematic review, meta-analysis.

Contributions of each author:

Author 1 - Gao-Feng Liu.

Email: lgftcm@163.com

Author 2 - Zhen Gao.

Email: gz0114@126.com

Author 3 - Zheng-Nan Liu.

Email: Izntcm@163.com

Author 4 - Min Yang.

Email: ymtcm89@163.com

Author 5 - Sheng Zhang.

Email: 517813211@qq.com

Author 6 - Tai-Peng Tan.

Email: tanyizhen12345@126.com 